\title{
Le syndrome de la poche à urines violettes
}

\section{Purple Urine Bag Syndrome}

\author{
N. Nieder $\cdot$ K. Habchi $\cdot$ T. Schmutz \\ Reçu le 26 mars 2017; accepté le 3 mai 2017 \\ (C) SFMU et Lavoisier SAS 2017
}

Une patiente âgée de 92 ans, grabataire, porteuse d'une sonde à demeure est adressée aux urgences pour une hyperthermie. L'urgentiste est d'emblée frappé par la coloration violette de la poche à urine alors que les urines vidangées sont jaune foncé mais non colorées. La biologie met en évidence une hyperleucocytose et un syndrome inflammatoire biologique marqué. La bandelette urinaire retrouve un $\mathrm{pH}$ urinaire à 9 , une leucocyturie sans nitrite et une protéinurie. Un examen cytobactériologique des urines est réalisé et une antibiothérapie par ceftriaxone est débutée. La patiente est hospitalisée en gériatrie.

Le Purple Urine Bag Syndrome (PUBS) est un syndrome curieux et rare, décrit pour la première fois en 1978 par Barlow. Il touche principalement les femmes âgées, alitées, constipées et sondées à demeure [1]. Comme son nom l'indique, il se caractérise par la coloration violette du collecteur à urine (Fig. 1). Cette teinte anormale peut surprendre les urgentistes et conduire à des examens inutiles. Elle s'explique par l'accumulation dans les urines d'un métabolite du tryptophane (contenu dans le bol alimentaire) : le 3-indoxysulfate. Dans certaines conditions ( $\mathrm{pH}$ urinaire alcalin, présence de certaines bactéries), ce 3-indoxysulfate urinaire se transforme en deux pigments (l'indigo de couleur bleue et l'indirubine de couleur rouge) qui se fixent sur le polychlorure de Vinyl qui constitue la sonde ou la poche à urine. Cette association pigmentaire entraîne la coloration violette du sac de vidange $[1,2]$. Outre le changement du collecteur à urine et le traitement d'une infection urinaire associée, le PUBS ne nécessite aucune thérapeutique ni investigation.

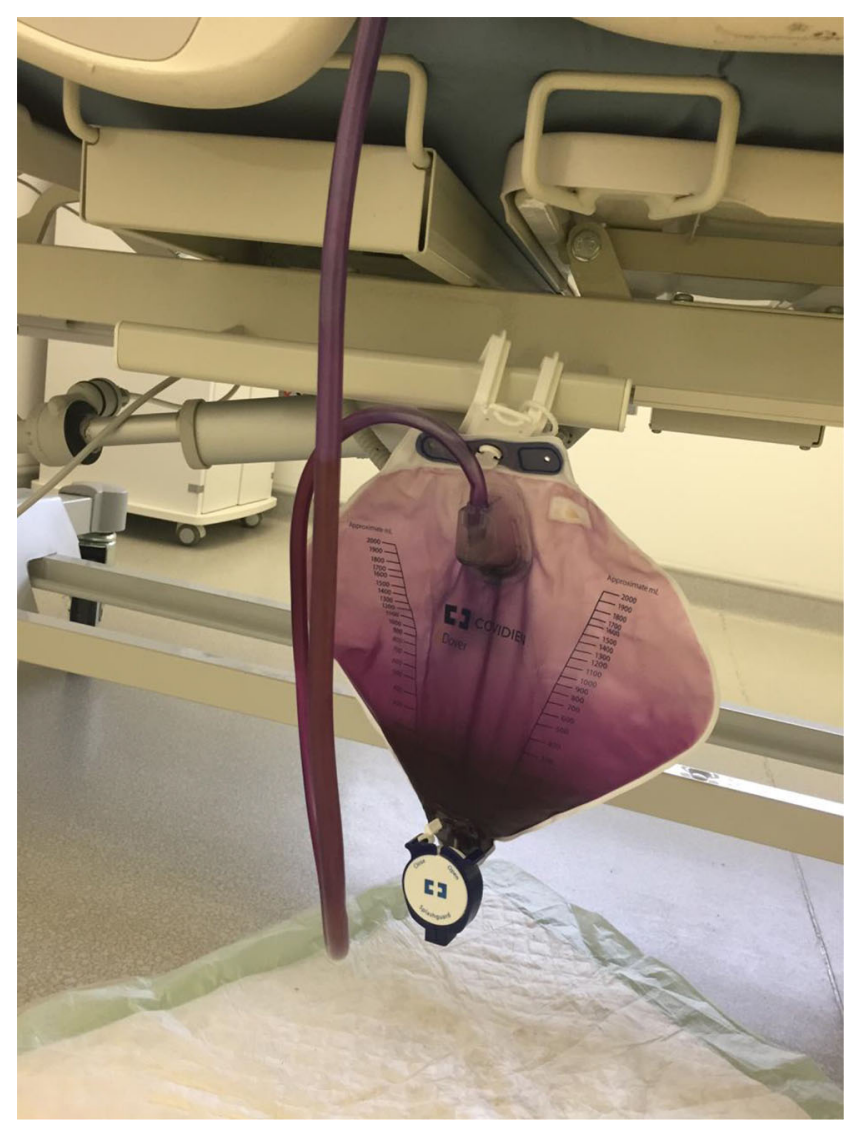

Fig. 1 La coloration violette du collecteur à urine signe le Purple Urine Bag Syndrome

\section{Références}

1. Mantani N, Ochiai H, Imanishi N, et al (2003) A case-control study of purple urine bag syndrome in geriatric wards. J Infect Chemother 9:53-7

2. Mumoli N, Vitale J, Brondi B, et al (2013) Purple urine-bag syndrome in a department of medicine. J Am Geriatr Soc 61:2240-1

N. Nieder $\cdot$ K. Habchi $\cdot$ T. Schmutz $(\bowtie)$

Structures de médecine d'urgence, hôpital de Mercy,

CHR Metz-Thionville, 1 allée du Château,

F-57085 Ars-Laquenexy, France

e-mail : t.schmutz@chr-metz-thionville.fr 